Research Article

\title{
Compact and High-Efficiency Rectenna for Wireless Power-Harvesting Applications
}

\author{
Dalia H. Sadek $\mathbb{D}^{1}{ }^{1}$ Heba A. Shawkey $\mathbb{D}^{2}{ }^{2}$ and Abdelhalim A. Zekry $\mathbb{D}^{1}$ \\ ${ }^{1}$ Faculty of Engineering, Ain Shams University, Cairo, Egypt \\ ${ }^{2}$ Electronics Research Institute (ERI), Giza, Egypt \\ Correspondence should be addressed to Dalia H. Sadek; doly_sadek@hotmail.com
}

Received 6 October 2021; Accepted 6 December 2021; Published 15 December 2021

Academic Editor: Gino Sorbello

Copyright $(92021$ Dalia H. Sadek et al. This is an open access article distributed under the Creative Commons Attribution License, which permits unrestricted use, distribution, and reproduction in any medium, provided the original work is properly cited.

A compact, single-layer microstrip rectenna for dedicated far-field RF wireless power-harvesting applications is presented. The proposed rectenna circuit configurations including multiband triple L-Arms patch antenna with diamond slot ground are designed to resonate at $10,13,17$, and $26 \mathrm{GHz}$ with $10 \mathrm{~dB}$ impedance bandwidths of $0.67,0.8,2.45$, and $4.3 \mathrm{GHz}$, respectively. Two rectifier designs have been fabricated and compared, a half wave rectifier with a shunted Schottky diode and a voltage doubler rectifier. The measured and simulated maximum conversion efficiencies of the rectifier using the shunted diode half-wave rectifier are $41 \%$, and $34 \%$, respectively, for $300 \Omega$ load resistance, whereas they amount to $50 \%$ and $43 \%$, respectively, for voltage doubler rectifier with $650 \Omega$ load resistance. Compared to the shunted rectifier circuit, it is significant to note that the voltage doubler rectifier circuit has higher efficiency. Both rectifier's circuits presented are tuned for a center frequency of $10 \mathrm{GHz}$ and implemented using $0.81 \mathrm{~mm}$ thick Rogers (RO4003c) substrate. The overall size of the antenna is $16.5 \times 16.5 \mathrm{~mm}^{2}$, and the shunted rectifier is only $13.3 \times 8.2 \mathrm{~mm}^{2}$ and $19.7 \times 7.4 \mathrm{~mm}^{2}$ for the voltage doubler rectifier. The antenna is designed and simulated using the CST Microwave Studio Suite (Computer Simulation Technology), while the complete rectenna is simulated using Agilent's ADS tool with good agreement for both simulation and measurements.

\section{Introduction}

Thanks to microsystems and wireless communications, wireless sensor networks (WSN) have expanded in many applications with energy independence as one of the fundamental challenges in the implementation of WSN in the real world. Recently, energy harvesting has become popular and wireless power harvesting devices look to be a promising technique for powering sensors' nodes that can be equipped with powering modules that can convert received electromagnetic energy into a DC current that can be utilized to charge the local battery. Rectenna, or "rectifying antenna," is the crucial component that determines the entire system performance. Different types of rectennas have been developed for high-power applications. The construction of energy conversion circuits to capture energy from a low power RF dedicated source at high frequencies has recently been the focus of study in wireless power transfer (WPT) [1].
In fact, the higher frequency bands provide a number of advantages, including smaller area, faster transmission rates, better signal penetration, and greater bandwidth [1]. The main challenges for building an efficient rectenna are low intercepted power levels, losses in the matching circuit, diode nonlinearity, and changes in circuit performance with input power level, input frequency, and termination load. All of these issues have been addressed in various literatures during the last few years. A lot of research has gone into developing high-efficiency rectennas. Most previous art designs, on the contrary, perform best at high input power, such as greater than $0 \mathrm{dBm}$ [2-5]. Zbitou et al., in [2], have designed an antenna array with efficiency $65 \%$ for $25 \mathrm{dBm}$ power input. In [3], Hagerty et al. designed a rectenna array, where 20 percent efficiency was reached for a power density of $62 \mu \mathrm{W} / \mathrm{cm}^{2}$ or equivalently, a power input of $13.27 \mathrm{dBm}$ (for rectenna geometric area of $\left(19 \times 23 \mathrm{~cm}^{2}\right)$. In [4], Falkenstein et al. designed a rectenna with efficiency of $54 \%$ 
which was obtained for power density $200 \mu \mathrm{W} / \mathrm{cm}^{2}$ or for $9.54 \mathrm{dBm}$ power input (assuming rectenna geometric area of $45 \mathrm{~cm}^{2}$ ). Finally, Tan and Liu designed a rectifier [5], with a measured conversion efficiency of $72 \%$ and length $10 \mathrm{~cm}$ at $10 \mathrm{GHz}$ with an input power of $87 \mathrm{~mW}(19.4 \mathrm{dBm})$. Highefficiency rectennas for low power input, such as less than $0 \mathrm{dBm}$, and low-loss substrates have also been the focus of many studies, where Costanzo et al. and Masotti et al. $[6,7]$ recommended increased efficiency even at a higher overall cost. A multiband rectenna was employed by Costanzo et al, at frequencies of $900 \mathrm{MHz}$ and $1750 \mathrm{MHz}$, achieving measured efficiency of $44.5 \%$ and $34.5 \%$, respectively, at input power of $-20.9 \mathrm{dBm}$ and $-13.7 \mathrm{dBm}$, respectively [6]. In [7], Masotti et al. introduced a rectenna operating at $900 \mathrm{MHz}$ with $33 \%$ efficiency for $-20 \mathrm{dBm}$ input power.

In this work, a Triple L-Arms microstrip patch antenna was designed [8] to achieve a high gain with small size at $5 \mathrm{G}$ bands of $10,13,17$, and $26 \mathrm{GHz}$. The antenna is small enough to be included in sensors, domestic equipment, and wearables. Also, it is appropriate for WPT and energy harvesting systems also; the design, simulation, and construction of two rectifiers' circuit topologies for microwave power transmission operating at the $10 \mathrm{GHz}$ band are presented. Then, the antenna and the rectifiers are combined to test and measure the complete rectenna. The highest efficiency has been achieved by careful selection of the diode used in the rectifier design and low-loss matching network design. The proposed rectenna can be used for low power integrated circuit as sensor nodes. Recent nanometer technologies has low threshold voltage, i.e., $45 \mathrm{~nm}$ CMOS technology has $0.6 \mathrm{~V}$ threshold voltage [8]. This paper is organized as follows. Section 2 introduces the antenna design and layout. Sections 3 and 4 present the shunt rectifier and voltage doubler design, respectively. Section 5 depicts the results of the rectenna. Finally, Section 6 concludes the work.

\section{Antenna Design and Layout}

The antenna is made with microstrip technology. It is described fully in [9], and here, we give an outline for it for the sake of completeness of the subject. Figure 1 depicts the geometry of the proposed Triple-L-Arms' patch slotted antenna (TLA).

The antenna is built on a ROGERS (RO4003c) substrate with $\varepsilon r=3.38$, thickness of $0.81 \mathrm{~mm}$, and $\tan =0.0026$ dissipation factor. The antenna is fed by a feeding line with a width of $W_{f}=2.1 \mathrm{~mm}$ and a length of $L_{f}=6.3 \mathrm{~mm}$. The TLA is then fabricated, and its photographs are shown in Figures 2(a) and 2(b).

Figure 3 demonstrates the return loss (S-parameter) value, as a result of antenna simulation and antenna measurement for sake of validation and comparison. The antenna operates at $10,13,17$, and $26 \mathrm{GHz}$ with a bandwidth of $0.67,0.8,2.45$, and $4.3 \mathrm{GHz}$, respectively. The wide bandwidth antennas are necessary to harvest power efficiently from the full spectrum. In this paper, the antenna is used for specific frequency at $10 \mathrm{GHz}$ not for wide range but for rectenna applications.

\section{Shunt Rectifier Circuit Architecture}

The single shunt diode topology was chosen for the rectifier circuit. The rectifier circuit's RF-to-DC conversion efficiency is maximized at an input power by applying a nonlinear optimization technique based on harmonic balance simulation and optimization goals. Figure 4 shows a general rectifier block diagram with three main sections: impendence matching for maximum power transfer between the antenna and the diode, a DC pass filter, and, finally, a DC load. The key aspects of a rectifier are the diode selection and the impedance matching network [10]. The SMS-7630 low barrier Schottky diode is chosen as the rectifying device. The junction potential of the diode is $0.34 \mathrm{~V}$ and break down voltage is $2 \mathrm{~V}$. Also, the leakage current of SMS-7630 diode is $1 E-4$ Ampere. The diode was efficient for low power operation due to their lesser junction potential [11]. The input impedance of a rectifier is a function of input power, load, and frequency because it is a nonlinear circuit. The nonlinear impedance of the rectifier circuit was examined in order to appropriately construct the matching network. The harmonic balance $(\mathrm{HB})$ simulator was used to evaluate the shunt rectifier in Keysight Advance Design System (ADS). The vendor's SPICE model of the diode SMS-7630 was imported into ADS.

Impedance matching network (IMN) can be accomplished in two ways: utilizing lumped LC components or using microstrip lines. For frequencies greater than $1 \mathrm{GHz}$, lumped components become lossy, and the insertion loss of the matching network becomes significant, lowering the rectifier's overall efficiency. In order to avoid these losses of lumped components, a transmission line-based IMN was used [12].

Two sections are responsible of the IMN; the first one is the transmission line-based impedance transformer which was designed to match the real input impedance to $50 \Omega$ in the desired band. (TL1) 1/8 $\lambda 0$ ( $\lambda 0$ represents the wavelength at $10 \mathrm{GHz}$ ), and the second one is butterfly stub. The imaginary input impedance was matched by inserting stubs (butterfly used to minimize the size of the rectifier). The overall network was optimized again in ADS to match the rectifier at the band for varying power levels. The layout of the designed microwave rectifying circuit is illustrated in Figure 5.

The proposed rectifying circuit is designed on Rogers (RO4003c) substrate with the relative dielectric constant $\varepsilon_{r}$ of 3.38, thickness $h$ of $0.81 \mathrm{~mm}$, and $\tan \sigma$ of 0.0026 . The geometric parameters of the rectifying circuit are listed in Table 1. Figure 6 shows the VNA (ROHDE\&SCHWARZ ZVA6) that is used to perform the return loss measurements. The simulated $\left|S_{11}\right|$ of the rectifying circuit in addition to measured results is shown in Figure 7. It is well matched at $10 \mathrm{GHz}$ with a minimum $\left|S_{11}\right|$ of $-14 \mathrm{~dB}$.

The difference between the simulation and measurement is generated by many parameters that are the parasitic parameters in the PCB and in the Schottky diode at high frequencies that can create higher parasitic impedance and higher error [13]. To reduce this deviation, the S-parameters for each SMT devices can be measured individually; extract 


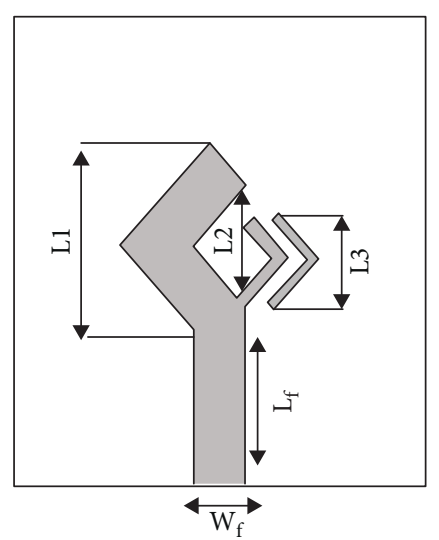

(a)

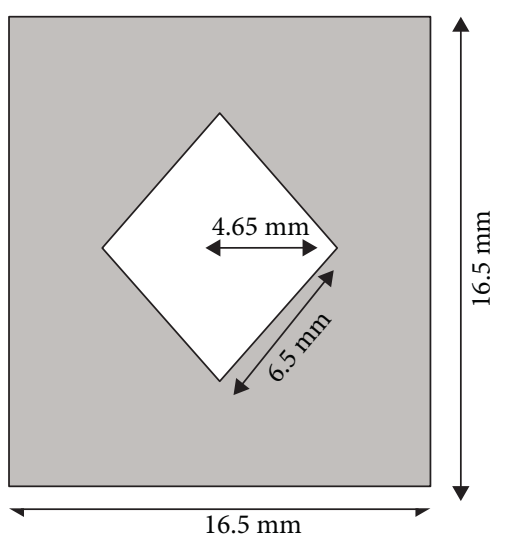

(b)

Figure 1: Configuration of the proposed antenna with dimensions. (a) Top view. (b) Bottom view.

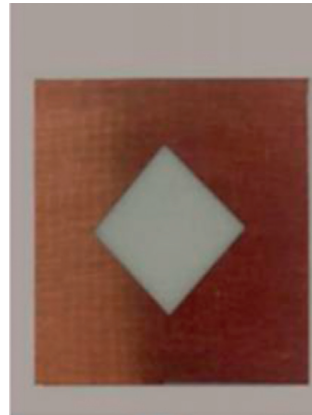

(a)

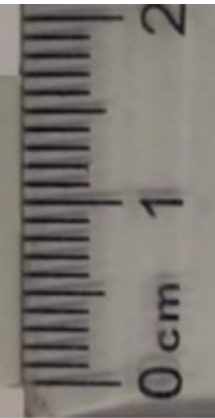

(b)
FIgURe 2: Photograph of the fabricated patch antenna: (a) bottom layer; (b) top layer.
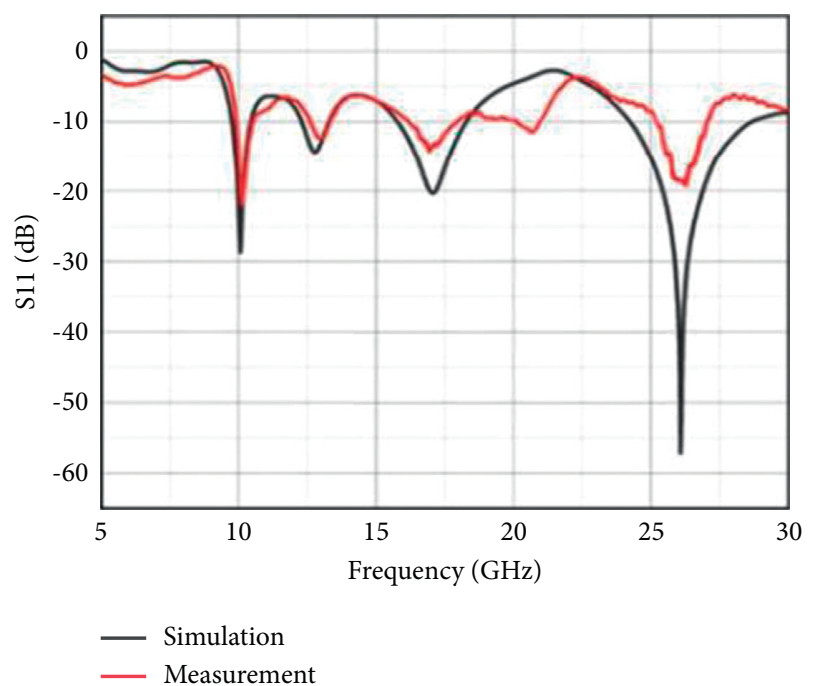

FIGURE 3: Comparison between simulation and measured reflection coefficient versus frequency.

their parasitics and use the extracted parameters in simulation. For narrow band operation, this solution will be accurate, but in wide band, the accuracy for VNA device decreases.

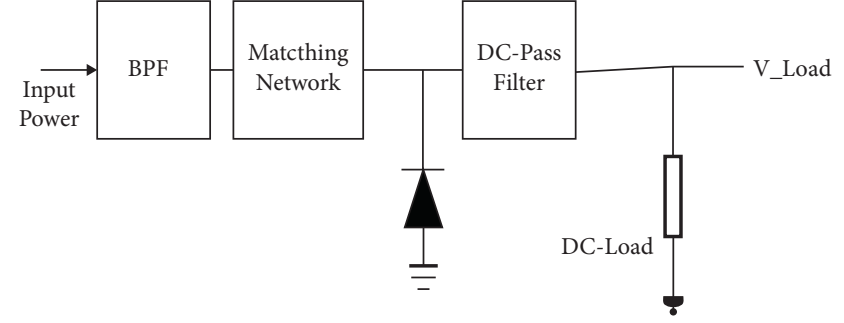

FIgURE 4: General rectifier block diagram.

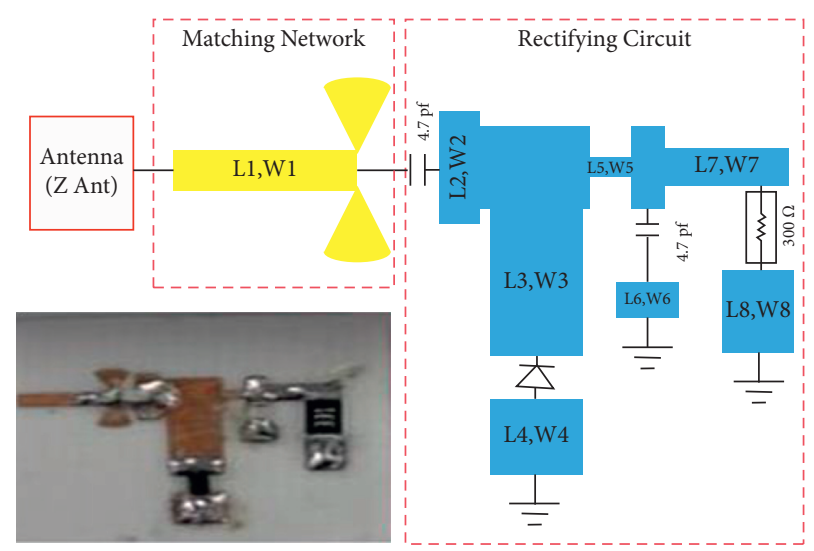

FIGURE 5: The shunt rectifier layout and photograph of the fabricated shunt rectifier.

TABle 1: Dimensions of the shunt rectifying circuit.

\begin{tabular}{lccc}
\hline & \multicolumn{3}{c}{ Geometrical parameters $(\mathrm{mm})$} \\
\hline L1 & W1 & L2 & W2 \\
4 & 0.8 & 1 & 3 \\
L3 & W3 & L4 & W4 \\
4 & 2.2 & 2 & 2.2 \\
L5 & W5 & L6 & W6 \\
1 & 0.5 & 1 & 1.5 \\
L7 & W7 & L8 & W8 \\
3 & 1 & 1.55 & 2.2 \\
\hline
\end{tabular}




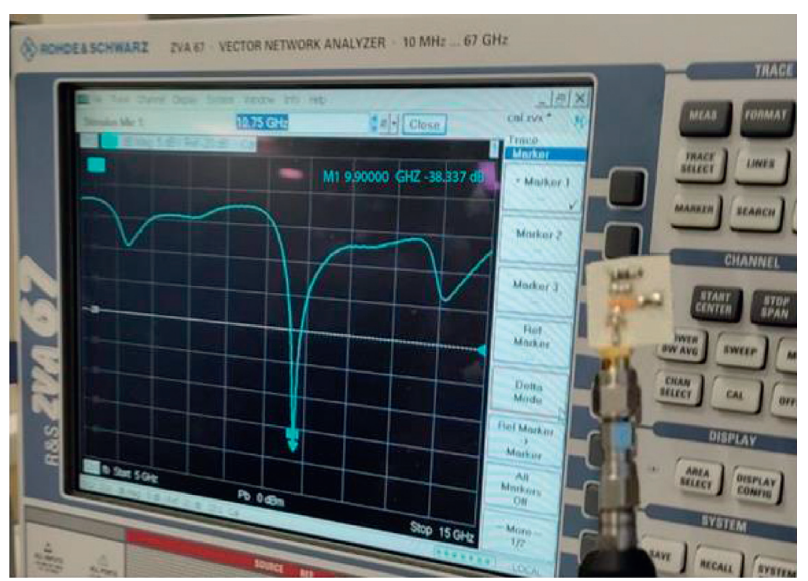

Figure 6: The measurement of the $S 11$ parameter using the VNA ROHDE\&SCHWARZ ZVA6.

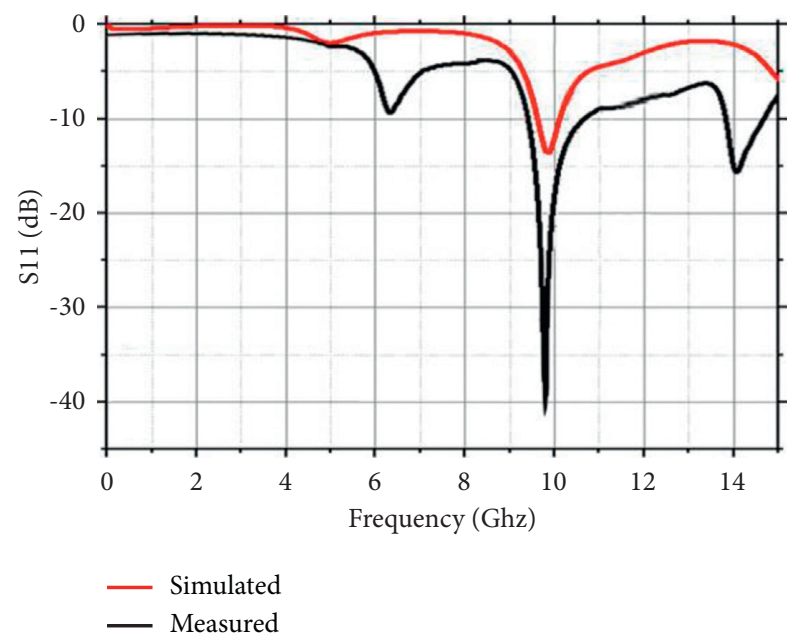

Figure 7: Comparison between simulation and measured reflection coefficient versus frequency.

As the deviations are due to tolerances, parasitics, and idealized models of the diodes, then their elaborate investigation will be undertaken in a future work. The rectifying circuit is fabricated and tested. The fabricated rectifying circuit is shown in Figure 5.

\section{Voltage Doubling Rectifier Circuit Architecture}

The voltage doubler rectifier (VDR) is a dual-diode amplitude-amplification circuit. The diode D1 and capacitor C1 make a peak rectifier, while the diode D2 and capacitor C2 make a voltage clamp [14]. VDR is a voltage doubler with a single stage that doubles the amplitude of input signals. In our design, a sms-7630 Schottky diode and its model with SOT-23 series pair.

Package with a voltage drop of $0.34 \mathrm{~V}$ was also employed in the voltage doubler rectifier. This voltage drop allows for faster switching and higher system efficiency. Figure 8 depicts the layout of a VDR. In microstrip, lines necessary for

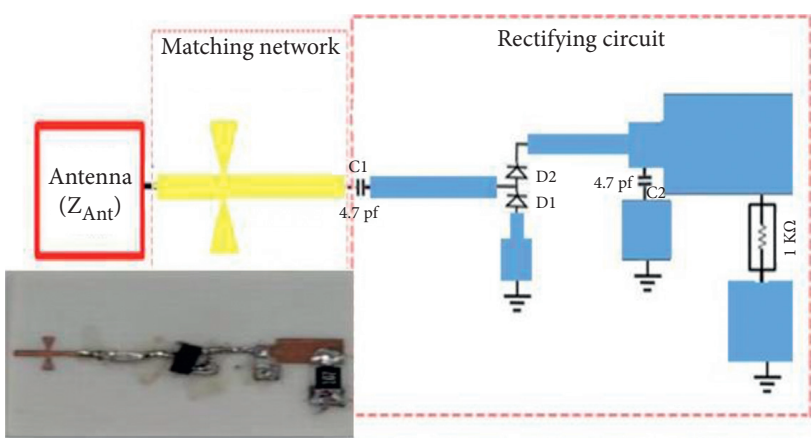

FIGURE 8: The layout of the voltage doubler rectifier and photograph of the fabricated voltage doubler rectifier.

efficient charging and impedance transformation are shorter. As a result, this design has the ability to reduce the amount of space allocated. The benefit of a smaller form factor is important because of a number of factors in a rectenna array, and rectifiers are required. The circuit was built, simulated, and optimized using Advance Design System (ADS) and then implemented on a Rogers (RO4003c) substrate.

The layout and fabrication of the voltage doubler rectifier are shown in Figure 8. The dimension of the rectifier system is $19.7 \times 7.4 \mathrm{~mm}^{2}$. The PCB (Printed Circuit Board) was manufactured in Electronics Research Institute (ERI) through many stages: drilling process using CNC machine, lamination process, photo-resist cladding, U.V. exposure after layout film fixing, chemical etching, and finally stripping stage.

\section{Complete Rectenna Measurements}

The receiving antenna (TLA), that is introduced in [9], and the rectifier are integrated on the same substrate and then measured. The measurement setup is shown in Figure 9. An Anritsu MG3697C RF/microwave signal generator is used to send a microwave signal which connected to a horn antenna with $12 \mathrm{dBi}$ gain at the proposed frequency $10 \mathrm{GHz}$. Then, a voltmeter is connected with the rectenna under test (RUT) to measure the DC output voltage for both rectennas. The antenna effective area is assessed when the antenna radiation properties are taken into account. As a result, the suggested rectenna's RF-DC conversion efficiency $(\eta)$ is expressed as

$$
\eta=\frac{V_{\mathrm{DC}}^{2}}{P_{\text {in }} R_{L}} \times 100 \%,
$$

where $V_{\mathrm{DC}}$ is the voltage across the resistive load $R_{L}$ and $P_{\text {in }}$ is the received RF input power. $P_{\text {in }}$ is defined in equation (2), where $P_{D}$ is the RF power density and $A_{\text {eff }}$ is the antenna effective area. $P_{D}$ and $A_{\text {eff }}$ are calculated in equations (3) and (4):

$$
\begin{gathered}
P_{D}=\frac{P t G t}{4 \pi r 2}, \\
A_{\text {eff }}=G_{r} \frac{\lambda}{4 \pi} .
\end{gathered}
$$

Pt (the transmitting power), Gt (the horn antenna gain), and $r$ (the distance between the transmitter and the 


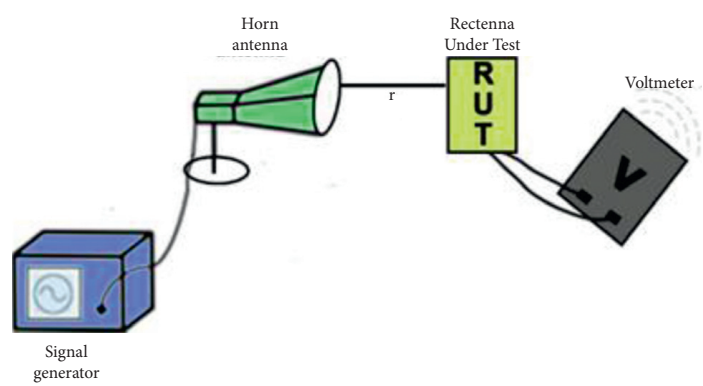

FIGURE 9: Rectenna measurement setup.

rectenna) all are known. Therefore, the RF-to-DC conversion efficiency can be measured. For far-field measurements, $r$ is chosen at a distance of $45 \mathrm{~cm}$ from the transmitting horn antenna, ensuring that both antennas were operating in their far-field zone with a reasonable degree of certainty. Figure 10 shows the photo of the measurement setup.

$$
P_{\text {in }}=P_{D} \times A_{\text {eff }},
$$

5.1. Measurement Results for Shunt Rectenna. The rectenna (antenna and shunt rectifier circuit) is tested over different input power levels. The simulated and measured conversion efficiencies and output DC voltage versus the input power for the rectenna are displayed in Figures 11(a) and 11(b), respectively. The performance parameters are the efficiency and the DC output voltage. The performance was measured by sweeping the input power (Pin) from $-10 \mathrm{dBm}$ to $10 \mathrm{dBm}$. This range of power is suitable for the application of RF wireless power transfer, Internet of Things (IoT) sensors, implantable devises, and wearable sensors in medical, healthcare, wellness, and sports applications, at $300 \mathrm{Ohms}$ of load resistance, taking into consideration the regulations for such sources in terms of maximum power that can be transmitted. These regulations, built to assure health and safety conditions while operating at ISM bands, are controlled by different associations such as the US Federal Communication Commission (FCC), the Canadian Standards Association (CSA), and the European Commission (CE). FCC: for example, they allow transmitting in ISM bands at a maximum power of $30 \mathrm{dBm}(1 \mathrm{~W})$, and for implantable devices, it does not exceed $40 \mathrm{~mW}$ [15]. The simulation results were plotted by red markers, and the measurement results were illustrated by black markers. The DC output can provide the maximum efficiency of $34 \%, 0.4 \mathrm{~V}$ output voltage at $2.5 \mathrm{dBm}$ of input power.

Figure 11 shows there is a peak value of the conversion efficiency at a specific input power pin. There is also a large discrepancy between the simulation results and the measured ones, which can be attributed to the model of the rectifier diode in addition to the fabrication tolerances of the geometrical features. However, the difference in DC output voltage between the measured and simulated results is much less pronounced.

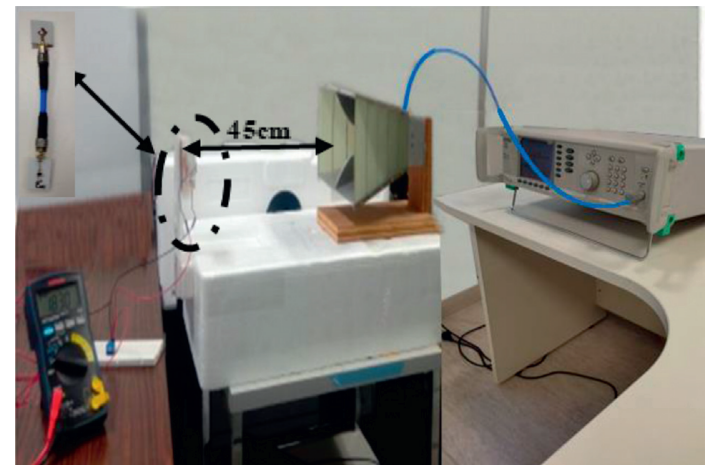

Figure 10: Photo of the measurement setup.

5.2. Measurement Results for Voltage Doubler Rectenna. The conversion efficiency of the rectenna (antenna and VDR) is measured with respect to the rectifier load at range of $-10 \mathrm{dBm}$ to $10 \mathrm{dBm}$ of input power. The optimal load resistance is around $650 \Omega$ for $5 \mathrm{dBm}$ input power at the frequency of $10 \mathrm{GHz}$. Figure $12(\mathrm{a})$ depicts the measured efficiency values. As a result, at $5 \mathrm{dBm}$ of input power, the maximum conversion efficiency of $43 \%$ is achieved. As can be observed in Figure 12(a), conversion efficiency increases until the input power approaches $5 \mathrm{dBm}$, beyond which it rapidly drops. The conversion efficiency reaches $38 \%$ between $-10 \mathrm{dBm}$ and $0 \mathrm{dBm}$ input power. Figure 12(b) shows that the output voltage has the values1V@5dBm,1.05V@7.5dBm, and 1.1 V@ $10 \mathrm{dBm}$, respectively. Another fundamental performance of rectenna circuits is the minimum input power to get the desired output power; the minimum power that can be detected by the proposed rectenna is $-10 \mathrm{dBm}$ and gives output voltage of $90 \mathrm{mV}$, whereas the minimum input power amounts to $3 \mathrm{dBm}$ to get the minimum desired output voltage of $0.8 \mathrm{~V}$. This value can be used in low power devices and low power operation in the CMOS threshold region. Compared to the first rectenna (shunt diode half wave rectifier), the size of the second rectenna (voltage double rectifier) is slightly larger, but it has higher efficiency and larger DC voltage.

At a frequency of $10 \mathrm{GHz}$ for all input power levels, the voltage doubler rectenna has a greater conversion efficiency and DC voltage than the shunt diode rectenna, as seen in Figures 11 and 12. However, the shunt rectifier has less complicated design and smaller area. As a result of this 


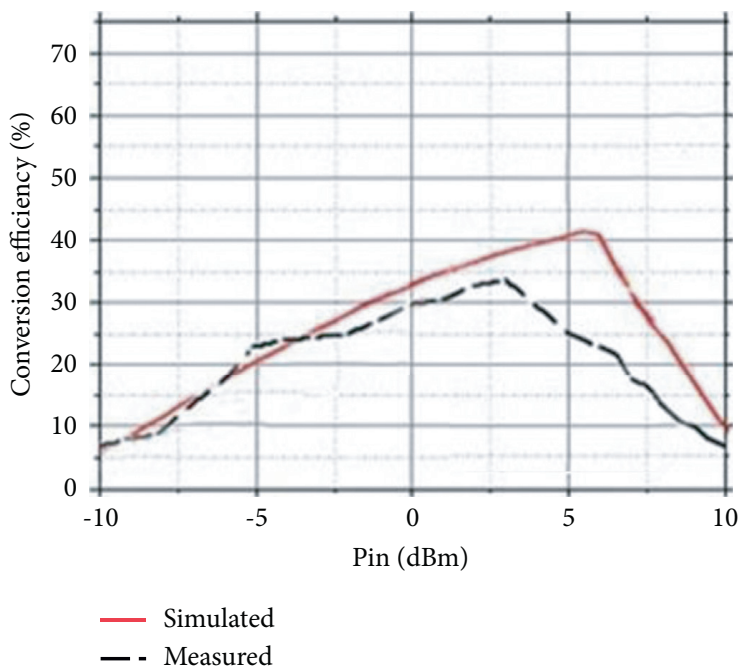

(a)

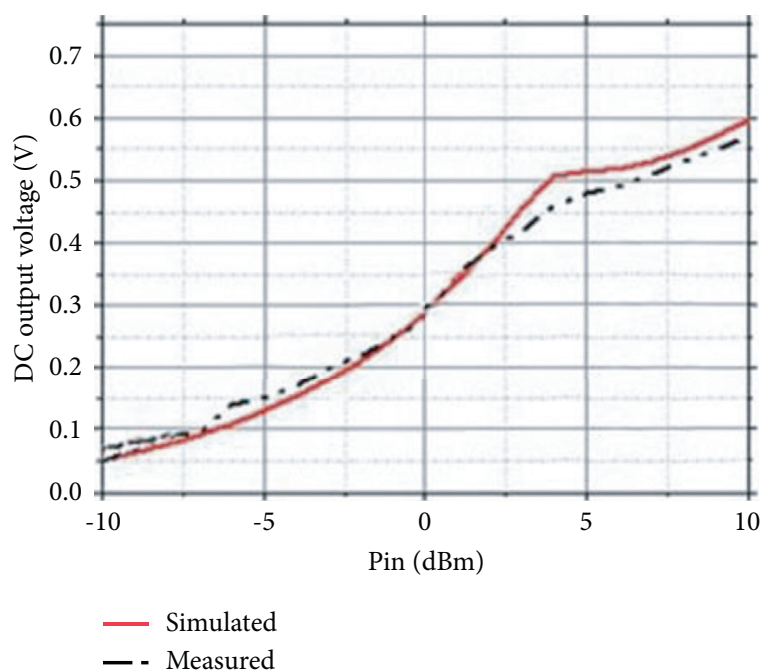

(b)

Figure 11: Measured and simulated of the rectenna with the voltage shunt rectifier versus continuous wave input power at $10 \mathrm{GHz}$. (a) Conversion efficiency. (b) Output DC voltage.

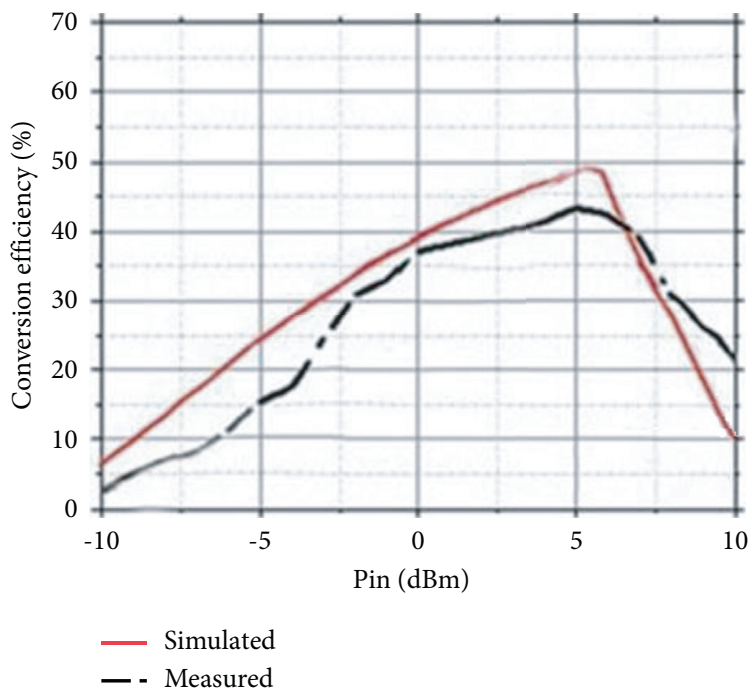

(a)

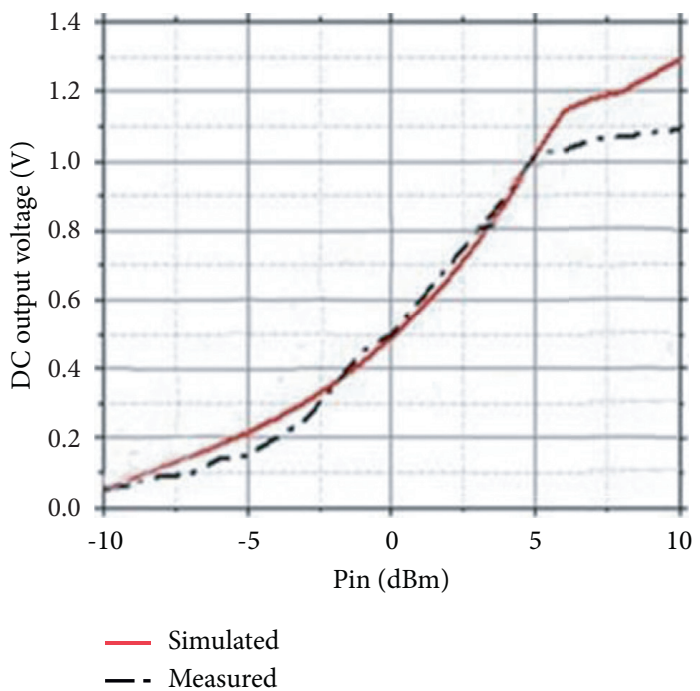

(b)

FIGURE 12: Measured and simulated of the rectenna with the voltage doubler rectifier versus continuous wave input power at $10 \mathrm{GHz}$. (a) Conversion efficiency. (b) Output DC voltage.

investigation, we have come to the following conclusion. When compared to the shunt diode rectenna, the voltage doubler rectenna performs better.

Figure 13 shows the measured conversion efficiency for the voltage doubler rectenna as a function of input powers with respect to different load resistance. Accordingly, the measured rectifying efficiency is less than $35 \%$ when the load resistance is between $100 \Omega$ to $400 \Omega$ for input power $6 \mathrm{dBm}$ and higher than $35 \%$ when the load resistance is bigger than $400 \Omega$.
Table 2 shows the performance comparison between the proposed rectenna and other previously reported in literature within the same frequency range. In [3], the maximum efficiency obtained from the rectenna is less than the proposed rectennas but with less needed power $(-16.7 \mathrm{dBm})$. The rectenna in [5] has more efficiency than the proposed rectenna but with more power input needed and more size area. The proposed rectennas (shunt and doubler) have more efficiency (30\% and 38\%), respectively, compared to [16] at the same power input $0 \mathrm{dBm}$. 


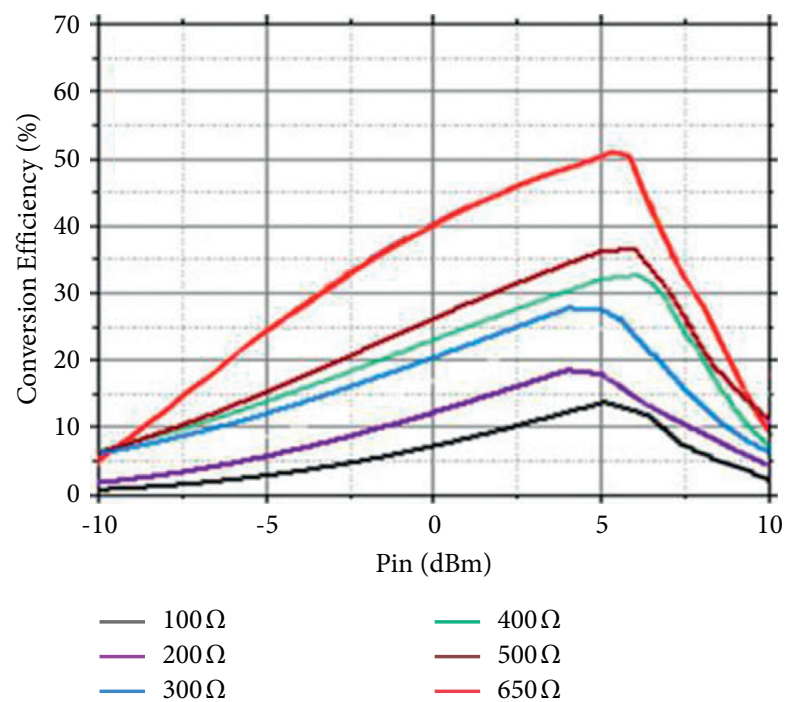

Figure 13: Conversion efficiency versus input power with respect to load resistances.

TABLE 2: RF-to-DC conversion efficiency vs. power input and frequency.

\begin{tabular}{lcccc}
\hline Reference & Max efficiency $(\%)$ & Power input $(\mathrm{dBm})$ & Rectenna size & Frequency $(\mathrm{GHz})$ \\
\hline$[3]$ & 20 & -16.7 & $19 \times 22 \mathrm{~mm}^{2}$ & $2-18$ \\
{$[5]$} & 72 & 19.4 & $10.5 \mathrm{~cm}^{2}$ & 10 \\
{$[16]$} & 25 & 0 & $1.5 \times 1.5 \mathrm{~cm}^{2}$ per unit cell & 10 \\
This work (shunt) & 34 & 7 & $13.3 \times 8.2 \mathrm{~mm}^{2}$ & 10 \\
This work (doubler) & 43 & 6 & $19.7 \times 7.4 \mathrm{~mm}^{2}$ & 10 \\
\hline
\end{tabular}

\section{Conclusion}

Rectennas with single shunt diode rectifier and doubler configurations are successfully developed and analyzed in this study. A low return loss microstrip patch antenna is employed as part of the receiving antenna and is integrated with the microstrip transmission line. A stub matching network is used for impedance matching between the antenna and rectifier sections. The rectenna produced gives enough output voltage to power any output load, such as sensors, unmanned vehicles, and LEDs. This rectenna circuit can also be used to dedicated far-field RF wireless power harvesting applications. An array of rectenna can be utilized to enhance the output voltage even more. We can additionally store the rectenna's output energy and use for charging purposes.

\section{Data Availability}

The data used to support the findings of this study are included within the article.

\section{Conflicts of Interest}

The authors declare that there are no conflicts of interest regarding the publication of this paper.

\section{Acknowledgments}

All fabrications and measurements were carried out in the Electronics Research Institute (ERI) labs.

\section{References}

[1] C. R. Valenta and G. D. Durgin, "Harvesting wireless power: survey of energy harvester conversion efficiency in far-field, wireless power transfer systems," IEEE Microwave Magazine, vol. 15, no. 4, pp. 108-120, 2014.

[2] J. Zbitou, M. Latrach, and S. Toutain, "Hybrid rectenna and monolithic integrated zero-bias microwave rectifier," IEEE Transactions on Microwave Theory and Techniques, vol. 54, no. 1, pp. 147-152, 2006.

[3] J. A. Hagerty, F. B. Helmbrecht, W. H. McCalpin, R. Zane, and Z. B. Popovic, "Recycling ambient microwave energy with broad-band rectenna arrays," IEEE Transactions on Microwave Theory and Techniques, vol. 52, no. 3, pp. 1014-1024, 2004.

[4] E. Falkenstein, M. Roberg, and Z. Popovic, "Low-power wireless power delivery," IEEE Transactions on Microwave Theory and Techniques, vol. 60, no. 7, pp. 2277-2286, 2012.

[5] F. Tan and C. Liu, "Theoretical and experimental development of a high-conversion-efficiency rectifier at X-band," International Journal of Microwave and Wireless Technologies, vol. 9, pp. 1-10, 2016.

[6] A. Costanzo, A. Romani, D. Masotti, N. Arbizzani, and V. Rizzoli, "RF/baseband co-design of switching receivers for multiband microwave energy harvesting," Sensors and Actuators A: Physical, vol. 179, pp. 158-168, 2012.

[7] D. Masotti, A. Costanzo, P. Francia, M. Filippi, and A. Romani, "A load-modulated rectifier for RF micropower harvesting with start-up strategies," IEEE Transactions on Microwave Theory and Techniques, vol. 62, no. 4, pp. 9941004, 2014 
[8] R. Nagulapalli, K. Hayatleh, S. Barker, S. Zourob, and A. Venkatareddy, "A novel current reference in $45 \mathrm{~nm} \mathrm{cmos}$ technology," in Proceedings of the 2017 Second International Conference on Electrical, Computer and Communication Technologies (ICECCT), Coimbatore, India, February 2017.

[9] D. Sadek, A. Zekry, and H. A. Shawkey, "Multiband triple L-arms patch antenna with diamond slot ground for $5 \mathrm{G}$ applications," The Applied Computational Electromagnetics Society Journal, vol. 36, no. 3, pp. 302-307, 2021.

[10] S. Ladan, A. B. Guntupalli, and K. Wu, "A high-efficiency $24 \mathrm{GHz}$ rectenna development towards millimeter-wave energy harvesting and wireless power transmission," IEEE Transactions on Circuits and Systems I: Regular Papers, vol. 61, no. 12, pp. 3358-3366, 2014.

[11] M. U. Rehman, W. Ahmed, and W. T. Khan, "High efficient dual band 2.45/5.58 rectifier for RF energy harvesting applications in ISM band," in Proceedings of the 2017 Asia Pacific Microwave Conference, IEEE, Kuala Lumpur, Malaysia, November 2017.

[12] M. U. Rehman, W. Ahmad, M. I. Qureshi, and W. T. Khan, "A highly efficient tri band (GSM1800, WiFi2400 and WiFi5000) rectifier for various radio frequency harvesting applications," in Proceedings of the Progress In Electromagnetics Research Symposium Journal, pp. 19-22, PIERS-FALL), Singapore, November 2017.

[13] K. Ekkaphol, Y. Zhao, and E. Leelarasmee, "A dual-band rectifier for RF energy harvesting systems," in Proceedings of the 11th International Conference on Electrical Engineering/ Electronics, May 2014.

[14] S. Riviere, F. Alicalapa, A. Douyere, and J.-D. Lan Sun Luk, "A compact rectenna device at low power level," Progress in Electromagnetics Research C, vol. 16, pp. 137-146, 2010.

[15] R. A. Bercich, D. R. Duffy, and P. P. Irazoqui, "Far-field RF powering of implantable devices: safety considerations," IEEE Transactions on Biomedical Engineering, vol. 60, no. 8, pp. 2107-2112, 2013.

[16] E. Kwiatkowski, C. T. Rodenbeck, T. Barton, and Z. Popovic, "Power-combined rectenna array for X-band wireless power transfer," in Proceedings of the 2020 MTT-S International Microwave Symposium, August 2020. 